\title{
BERN RADIOCARBON DATES V
}

\author{
H. OESCHGER and T. RIESEN
}

Physikalisches Institut, Universität Bern

This list includes about half of the samples measured during the last year. Many studies need further investigation and the results will be published later when additional information will make better interpretations possible.

The equipment and the technique is essentially the same as described earlier (Bern IV).

Reliable results on $\mathrm{CO}_{2}$ samples extracted out of ice are obtained with the small counter mentioned in Bern IV.

Our laboratory is financed by the Schweizerischer Nationalfonds. The authors wish to thank H. Loosli and P. Horisberger for their assistance in the measurements of part of the samples. They also thank M. Welten and H.-G. Bandi for their help in selecting and discussing the samples.

USA CRREL, the University of Bern and the Swiss Glacier Commission conducted a joint research project during March-April 1964 in the Tuto Tunnel, North Greenland, which runs $350 \mathrm{~m}$ horizontally into the polar ice sheet (Langway et al., 1965). Four samples were taken at each of two locations, approximately $300 \mathrm{~m}$ (Location 1) and $200 \mathrm{~m}$ (Location 2) from the tunnel portal. For the extraction two completely independent methods were used: precipitation in sodium hydroxide, and collection in molecular sieve (Oeschger et al., 1965).

The samples from Location 1 measured so far give ages of 2500 to 3000 B.P., whereas for Location 2 dates of 5000 B.P. to 6000 B.P. are obtained. The final results will be published after the measurement of all samples and the evaluation of the other studies connected with this project.

\section{Murifeld-Bern series, Switzerland}

Gyttja and peat from lake and bog deposit, Murifeld, near Bern (46 56 22" Lat, $7^{\circ} 28^{\prime} 35^{\prime \prime}$ E Long). Coll. 1962 and subm. by M. Welten, Univ. of Bern. Comment (M.W.): deep excavations in connection with construction of a highway permitted taking of samples on open walls of sediments, already well-known by pollen-analyses. The clearly developed late-glacial sediments proved to contain sufficient organic material for sample $\mathrm{C}^{14}$ dating. Location was first in Switzerland where Alleröd was dated (B-38, Bern I). It is now the first to record the Bölling-zone: the pollen diagram is clear and the dates fix the warm time as beginning at ca. 11,400 B.C., and ending at ca. 10,500 B.C. in full correspondence with north and central European datings. The oldest date (B-684) fixes a definite stage in establishment of late-glacial vegetation 
at this location. Another sample of Bölling age in Switzerland Lobsigensee (B-398, Bern IV), though not proved to belong to the zone, is fully confirmed by this series. B-501 was measured twice; being more than one thousand years too old, it can only be supposed that stratification was disturbed, possibly when dead-ice melted off.

B-439. Murifeld, $281 \mathrm{~cm}$ depth

$10,580 \pm 120$

8630 B.C.

B-440. Murifeld, $291 \mathrm{~cm}$ depth

$10,580 \pm 200$

8630 B.C.

B-441. Murifeld, $429 \mathrm{~cm}$ depth

$13,210 \pm 400$

Samples from a pollen profile excavated a lew years ago.

B-501. Murifeld, $69 \mathrm{~cm}$ above horizon of reference

B-500. Murifeld, $35 \mathrm{~cm}$ above horizon of reference

B-499. Murifeld, $19.5 \mathrm{~cm}$ above horizon of reference

B-497. Murifeld, $5.5 \mathrm{~cm}$ above horizon of reference

B-683. Murifeld, $15 \mathrm{~cm}$ below horizon of reference

B-684. Murifeld, $42 \mathrm{~cm}$ below horizon of reference

$$
\begin{gathered}
11,580 \pm 200 \\
9630 \text { в.с. } \\
11,360 \pm 200 \\
9410 \text { в.с. }
\end{gathered}
$$

$11,900 \pm 200$ 9950 B.C.

$12,730 \pm 200$

10,780 в.c.

$13,340 \pm 200$

11,390 в.C.

$13,860 \pm 200$

11,910 B.c.

\section{Mont Carré series, Switzerland}

Peat and gyttja from Mont Carré, small bog in Val d'Hérémence, Valais $\left(46^{\circ} 9^{\prime} 11^{\prime \prime} \mathrm{N}\right.$ Lat, $7^{\circ} 22^{\prime} 12^{\prime \prime}$ E Long, alt $\left.2290 \mathrm{~m}\right)$. Coll. Aug. 1964 and subm. by M. Welten. Comment (M.W.): the 3 samples give an approx. idea of the chief period of development of Hypnaceae-bog situated high above actual timberline. In this case organic matter originates from Atlantic and subboreal time. Within this period accumulation of moss-turf was ca. $0.33 \mathrm{~mm} / \mathrm{yr}$. Vegetational history is recorded palynologically.

B-627. Mont Carré, $63 \mathrm{~cm}$ depth

B-628. Mont Carré, $88 \mathrm{~cm}$ depth

B-629. Mont Carré, 140 em depth
$4800 \pm 120$

2850 B.C.

$5260 \pm 120$

3310 B.c.

$6740 \pm 150$

4790 в.c. 


\section{Gondo-Alpje series, Switzerland}

Peat and gyttja from Gondo-Alpje, Valais $\left(46^{\circ} 12^{\prime} 30^{\prime \prime} \mathrm{N}\right.$ Lat, $8^{\circ} 6^{\prime}$ $50^{\prime \prime}$ E Long, alt $\left.1635 \mathrm{~m}\right)$. Coll. Sept. 1965 and subm. by M. Welten. Comment (M.W.): the 5 samples date vegetational history and different sand-layers, thought to be climatological indices on southern slope of Alps.

\section{B-699. Gondo-Alpje, $77 \mathrm{~cm}$ depth}

B-630. Gondo-Alpje, $192 \mathrm{~cm}$ depth

B-631. Gondo-Alpje, $287 \mathrm{~cm}$ depth

B-632. Gondo-Alpje, $387 \mathrm{~cm}$ depth

B-633. Gondo-Alpje, $430 \mathrm{~cm}$ depth

$$
1400 \pm 100
$$
A.D. 550

$3060 \pm 120$

1110 B.c.

$3740 \pm 120$

1790 B.C.

$4670 \pm 130$

2720 B.c.

$5310 \pm 200$

3360 B.C.

\section{Simplon-Hopschensee series, Switzerland}

Samples of gyttja and clay-gyttja were taken by boring with Hillersampler at Simplon-Hopschensee, Valais $\left(46^{\circ} 15^{\prime} 12^{\prime \prime} \mathrm{N}\right.$ Lat, $8^{\circ} \mathrm{l}^{\prime} 25^{\prime \prime} \mathrm{E}$ Long, alt $2018 \mathrm{~m}$ ). Coll. Oct. 1963 by M Welten and K. Heeb; subm. by M. Welten. Comment (M.W.): investigations over two years with repeated sampling and remeasuring have convincingly confirmed existence of a layer of Alleröd-mud at the extremely high altitude of $2017 \mathrm{~m}$ above sealevel. Up to now the highest altitudes where Alleröd was clearly proved by pollen-analysis were not much above $1500 \mathrm{~m}$, most higher localities having too little organic matter for $\mathrm{C}^{14}$-dating and yielding only clay and boulder material during late-glacial period. Result seems to fall in Bölling-period, but the pollen diagram is against this interpretation. Considering the standard deviation of B-608, an Alleröd date is not ruled out. Other results date stages of vegetational history to be discussed elsewhere. B-636 proved an early invasion-peak of spruce in the pollen diagram to be a mistake, resulting from pollen contamination during boring.

B-634. Simplon-Hopschensee, $97 \mathrm{~cm}$ depth

B-669. Simplon-Hopschensee, $201 \mathrm{~cm}$ depth

B-635. Simplon-Hopschensee, $246 \mathrm{~cm}$ depth

B-635E. Simplon-Hopschensee, $277 \mathrm{~cm}$ depth
$660 \pm 80$
A.D. 1290
$3230 \pm 100$
1280 B.C.
$4500 \pm 300$
2550 B.C.
$5040 \pm 150$
3090 в.C.


B-636. Simplon-Hopschensee, $328 \mathrm{~cm}$ depth

$3970 \pm 120$

2020 B.C.

B-610. Simplon-Hopschensee

$7730 \pm 180$

5780 B.c.

Gyttja, $22.5 \mathrm{~cm}$ above boulders and clay of Younger-Dryas-period.

\section{B-609. Simplon-Hopschensee}

$9000 \pm 150$

Gyttja, $7.5 \mathrm{~cm}$ above the boulder-and-clay layer.

\section{B-530. Simplon-Hopschensee}

7050 B.C.

Gyttja, $5 \mathrm{~m}$ above the boulder-and-clay layer.

$9530 \pm 250$

7580 B.C.

\section{B-608. Simplon-Hopschensee}

$12,580 \pm 200$

$15 \mathrm{~cm}$ of clay-gyttja under the $70-\mathrm{cm}$ thick layer of boulders and clay supposed to originate from Younger-Dryas-period.

\section{Hellelen-Zeneggen series, Switzerland}

Peat and gyttja from peat-basin at Hellelen-Zeneggen, Valais $\left(46^{\circ}\right.$ $17^{\prime} 3^{\prime \prime} \mathrm{N}$ Lat, $7^{\circ} 50^{\prime} 40^{\prime \prime}$ E Long, alt $1510 \mathrm{~m}$ ). Coll. April 1965 and subm. by M. Welten. Comment (M.W.): series dates a remarkable sequence of organic sediments covering the whole postglacial period. Rate of sedimentation for gyttja between 6000 and 2000 B.c. reached $0.33 \mathrm{~mm} / \mathrm{yr}$, whereas between 1000 B.C. and A.D. $14001.6 \mathrm{~mm}$ of sedge-peat was formed each year. Consequently period of culture from Bronze Age to late Middle Ages is very well developed in pollen diagram. Details will be published later.

B-637. Hellelen-Zeneggen, $113 \mathrm{~cm}$ depth

B-724. Hellelen-Zeneggen, $210 \mathrm{~cm}$ depth

B-638. Hellelen-Zeneggen, $270 \mathrm{~cm}$ depth

B-639. Hellelen-Zeneggen, $438 \mathrm{~cm}$ depth

B-640. Hellelen-Zeneggen, $480 \mathrm{~cm}$ depth

B-723. Hellelen-Zeneggen, $562 \mathrm{~cm}$

B-641. Hellelen-Zeneggen, $622 \mathrm{~cm}$ depth
$1200 \pm 100$ A.D. 750

$1775 \pm 100$ A.D. 175

$2320 \pm 100$

370 B.c.

$3120 \pm 120$

1170 B.C.

$3920 \pm 100$

1970 B.c.

$5970 \pm 120$

4020 B.C.

$8160 \pm 130$

6210 B.C. 


\section{Krauchtal series, Glarus, Switzerland}

Wood fragments found in deposits from a landslide during technical borings near Krauchtal $\left(46^{\circ} 58^{\prime} 32^{\prime \prime}\right.$ N Lat, $9^{\circ} 13^{\prime} 34^{\prime \prime}$ E Long, alt 1400 m). Coll. and subm. Nov. 1964 by Chr. Schaerer and Crettaz, ETH, Zürich.

\section{B-660. Krauchtal}

From uppermost part of landslide deposit.

\section{B-661. Krauchtal}

From an under part of landslide deposit.
$3560 \pm 120$

1610 B.C.

$6395 \pm 150$

4445 B.C.

\section{Robiei series, Val Bavona, Switzerland}

Fragments of trees found during excavation work in a peat deposit above present timberline at Robiei, Val Bavona, Ticino $\left(46^{\circ} 26^{\prime} 40^{\prime \prime} \mathrm{N}\right.$ Lat, $8^{\circ} 30^{\prime} 56^{\prime \prime} \mathrm{E}$ Long, alt $\left.1880 \mathrm{~m}\right)$. Coll. 1964 and subm. by Maggia Kraftwerke, Locarno.

\section{B-675a. Trunk (Larix decidua)}

\section{B-675b. Bough (Pinus cembra)}

\section{Basel series, Pfalz, Switzerland}

Bones from tombs found on the Pfalz behind the minster of Basle $\left(47^{\circ} 34^{\prime} \mathrm{N}\right.$ Lat, $7^{\circ} 35^{\prime} \mathrm{E}$ Long). Coll. August 1965 and subm. by R. Moosbrugger, Basle. Comment (R.W.): $\mathrm{C}^{14}$ ages show that tombs are contemporary or a little older than adjacent Carolingian crypt from beginning of 9 th century A.D.

\section{B-681. Tomb 2}

\section{B-682. Tomb 2, "Vorbestattung"}

$1180 \pm 120$

\section{A.D. 770}

$1310 \pm 100$

Date lists:

REFERENCFS

Bern I Oeschger, Schwarz and Gfeller, 1959

Bern IV Oeschger and Riesen, 1965

Langway, C. C., Jun., Oeschger, H., Renaud, A., Alder, B., 1965, Sampling polar ice for radiocarbon dating: Nature, v. 206, p. 500.

Oeschger, H., Alder, B., Loosli, H., 1965, Radiocarbon dating of ice: Proceedings of the C14- and Tritium-Conference, Washington State Univ., Pullman.

Oeschger, H., Schwarz, U., Gfeller, Chr., 1959, Bern radiocarbon dates I: Am. Jour. Sci. Radioc. Supp., v. 1, p. 133-143.

Oeschger, H., Riesen, T., 1965, Bern radiocarbon dates IV: Radiocarbon, v. 7, p. 1-9. 